\title{
An Improved Grey Wolf Optimization Algorithm for Liquid flow Control System
}

\author{
Pijush Dutta \\ Department of Electronics \& Communication Engineering,Global Institute of Management \& Technology \\ Krishnagar ,India, Nadia :741102 \\ Email: pijushdutta009@gmail.com \\ Madhurima Majumder \\ Department of Electrical \& Electronics Engineering, Mirmadan Mohanlal Government Polytechnic, Gobindapur, \\ Plassey, West Bengal 741156 \\ Email: madhurimam128@gmail.com
}

\section{Asok Kumar}

Dean of student welfare Department, Vidyasagar University, Medinipur, West Bengal, India, Pin: 713305, Email: asok_km650@rediffmail.com

Received: 22 April 2021; Accepted: 12 June 2021; Published: 08 August 2021

\begin{abstract}
Liquid flow in a process industry is one of the significant factors which should be controlled to get the better quality and decrease the expense of generation. Customary methodology includes manual tuning of the input process parameter to obtain the required flow rate is tedious and exorbitant. Notwithstanding, estimation of a precise computational model for fluid stream control procedure can fill in as elective methodology. It is only a non-straight enhancement issue. As a contextual investigation, the WFT - 20-I measure control arrangement for flow rate measurement and Control issue is thought of. In this work we proposes a hybrid improved particle swarm optimization (PSO-GWO) used to start the people's position, which can build the decent variety of the wolf pack, balance the global and neighborhood search capacity of the calculation and improve the intermingling pace of the calculation contrast with the Gray wolf enhancement (GWO) and Particle swarm advancement (PSO). Non linear models are improved utilizing those recently proposed streamlining strategies. Additionally all the utilized optimization techniques can anticipate the fluid stream rate with good exactness. The outcomes were investigated by utilizing the root mean square error (RMSE), exactness, and the different measures to evaluate the level of identification performance of the liquid flow contextual analysis model. The trustworthiness of the present models was compared with the past model for similar subsystems utilizing competitive intelligent methodologies. The measurable examination of the acquired outcomes produced the proposed HPSOGWO has most elevated generally speaking proficiency (i.e.99.96\%) and it beat the others strategies for the majority of the instances of demonstrating for fluid stream control process. The outcomes of the present model show that the proposed approach gives prevalent demonstrating execution and outflanks its rivals.
\end{abstract}

Index Terms: Liquid flow process, Grey wolf optimizer, Particle swarm algorithm, hybrid algorithm.

\section{Introduction}

Typically, in a fluid stream control process, stream rate relies upon a few significant components like sensor yield, pipe diameter, fluid conductivity, fluid consistency and so forth[1,2]. In present research an advance scientific model is created so that it can portray the liquid flow control process in effective way.

Customarily, Flow rate is determined by utilizing by different sensor like anemometer [3], ultrasonic sensor [4], thermal sensor [5] and so forth. Because of high estimating precision and extremely short reaction time, anemometer type warm stream sensor has been broadly utilized in aviation, petroleum processing plant, flammable gas and so forth for standard estimation.

Several studies performed in this field some of which are: an enhanced Artificial Neural Network model [6] proposed to decide the speed of the gas concerning test information like sensor voltage and the liquid temperature. An enhanced neural system model for an ultrasonic sensor where the flow rate depends upon the potential input parameters [7]. Except for the ANN nonlinear model a few nonlinear models like Regression Analysis [8], Response Surface Methods [9], Analysis of Variance (ANOVA) [10], and so on are well known where polynomial, strategic, quadratic, 
exponential, logarithmic, power and so forth also used in the field of process control industry to make a connection between process output, flowrate \& independent input process variables like pipe diameter \& liquid nature, etc. conditions can be utilized to speak to the conduct of a framework [11]. During a mathematical extraction strategy, a proficient improvement procedure is needed to streamline the model boundaries to such an extent that exploratory yield fits best with the re-enacted yield. Thusly, precise demonstrating of fluid stream control measure is a regular case of nonlinear improvement issues where we have to distinguish ideal estimations of the model boundaries. The exactness of the separated boundaries relies upon the choice of an appropriate streamlining method.

Fuzzy logic controller based intelligent control technique has been used to anticipate the stream rate in fluid flow process framework [12]. Here author applied a distinctive types $\&$ nature of membership function of a input $\&$ response variables to design the effective model. From the investigation, it was seen that the proposed model achieved least RMSE of $7.98 \%$. Artificial neural network model (ANN) has been used for forecast of fluid stream rate going through the flow sensor in a fluid stream process[13]. A NN model was created misusing trial estimations of liquid flow. The neural system model was prepared and tried utilizing MATLAB tool. The outcomes anticipated from ANN model was contrasted and trial estimations. Examination shows that the most extreme RMSE is 2.94\%. SVM and KNN algorithms has been proposed to classify the datasets and get expectation (find shrouded designs) for target in liquid flow process [14]. The framework finds the data pattern to foresee future informational collections. A hybrid GA-optimized ANN model has been examined for the liquid flow process [15]. From the numerical outcomes, it was seen that rank choice hybrid GA-ANN model is superior with respect to other choices: Tournament and Roulette wheel \& it produced the exactness of $98.42 \%$. Optimized ANFIS model has been proposed for the Prediction of the liquid flow in a liquid flow control process [16]. From the result it has been seen that best ANFIS structure provides 2.143\% of RMSE and $0.504 \%$ of MAE respectively. ANN optimized flower pollination algorithm (FPA) has been utilized to improve the model precision and convergence speed (Dutta \& Kumar, 2019). Result shows the accuracy about 94.17\%. Three diverse improved variants of unique elephant swarm water search calculation (ESWSA) for displaying of fluid stream control process and tried against the present issue to advanced the coefficient of the parameter from ANOVA and Response surface technique[17]. From the outcome investigation it is seen that all calculations and models can foresee the fluid stream rate with palatable precision for example generally speaking proficiency is about $40 \%$.

A metaheuristic is one of the most mainstream subclass enhancement strategies where advancement measures are normally motivated by physical wonders, creatures' practices, or developmental ideas[1,18]. Swarm Intelligence (SI) based strategies is one of the famous \& effective subclasses of metaheuristic optimization. In SI it follows the social conduct of multitudes, groups, runs, or schools of creepy crawlies and creatures in nature where the inquiry operators explore utilizing the re-enacted group and social knowledge of animals [19]. Straightforwardness, adaptability, inference free component and neighborhood optima evasion capacity are the principal explanations for the prevalence of metaheuristics. These qualities make metaheuristics enormously fitting for genuine enhancement issues. There are several research conducted on the basis swarm intelligence those is Particle swarm improvement (PSO) [20], Bat Algorithm (BA) [21], Artificial Bee Colony (ABC) [22], Firefly Algorithm (FA)[23], Flower Pollination Algorithm (FPA)[24], Ant Colony Optimization (ACO)[25], Cuckoo Search optimization(CSO) [26], Elephant swarm water search algorithm(ESWSA)[27,28], Simulated Annealing [29], Sine Cosine Optimization [30], Salp Swarm Algorithm[31] and so on. For improving better computational time, precision, intermingling speed, better investigation, proper parameters tuning and misuse capacity a single metaheuristic is not appropriate for taking care of all this, so a metaheuristics use to change the existing one, is known as the No Free Lunch hypothesis [32].

The GWO calculation is right off the bat proposed by the [33] the calculation applies the chasing procedure pursued by the dim wolves, after that numerous changes have been proposed to beat the inadequacies of the calculation. [34] proposed the utilization of a period shifting capacity of diminishing directly for changing the estimation of vector $a$ which adjusts the investigation and misuse capacities of the GWO. Besides the valid statement set strategy is utilized for creating the underlying populace which improves the worldwide union of the calculation. [35] exhibited a hybrid DE-GWO which uses to refresh the past best places of Alpha, Beta and Delta wolves in such way makes GWO inclined to the stagnation. Another alteration of GWO is proposed [36] which change the position update condition of standard GWO calculation. The introduced adjustment utilizes the mean of wolf position vectors for the estimation of development course of wolves. The utilization of exponential capacity for the rotting the estimation of vector $a$ is displayed [34] \& utilization of exponential root work improves the abuse and investigation capacity of the calculation. A Genetic Algorithm (GA) based beginning populace age approach for GWO is displayed by [37], the correct introduction prompts more prominent plausibility in finding worldwide ideal. Similarly as with other meta-heuristic calculations the GSO likewise requires legitimate starting worth settings of factors to accomplish the best outcomes. Since these qualities relies on issue viable and must be evaluated based on target work attributes to address this issue [38] exhibited fortification learning and neural system based methodology EGWO (Experienced GWO) which assesses the correct parameters esteems for the calculation. In their model the investigation pace of each wolf assessed by wolves own understanding and the momentum condition of the hunt space. The experience is put away as neural system that maps specialist states to comparing activities. The Powell nearby streamlining based GWO calculation PGWO is displayed [39]. 
Because of more noteworthy possibility of being stuck in the neighborhood least arrangement, they are presently being subbed by the utilizations of different metaheuristic calculations which would give the close to ideal or worldwide ideal arrangements in less time due their high convergence speed. In this study, we proposed three different optimization algorithms PSO, GWO \& HPSOGWO for modeling of nonlinear liquid flow control process. In this paper the following section are structured as follows. Section 2 represents the set up of the control process. Section 3 represents the formulation of mathematical model by ANOVA, optimization techniques \& their methodology. Section 4 described simulated results analysis followed by conclusions in final sections.

\section{Presentation \& Modeling of the Liquid Flow Control Process}

Present research performed with (Model no WFT-20-I) help of Flow \& level measurement \& control set up [40]. Contact type flow sensor (anemometer), PVC pipe, Rotameter \& $31 / 2$ Digital Multimeter is used as external components to perform the experiment. For this research total 117 sample data has been taken to train the model while 17 datasets are utilized for the testing purpose [40]. Datasets consist of three independent variables sensor output voltage, pipe diameter \& liquid (water) conductivity and one dependent variable: flow rate shown in Table 3 . Name of process input $\&$ output parameters along with range considered for optimized the flow rate using metaheuristic optimization technique.

The experimental set up for the resent research shown in Figure 1. In this inspection, the liquid velocities measured were in the range $01 \mathrm{pm}-600 \mathrm{lpm}$. When this liquid passing through the semiconductor based flow sensor it provides the voltage in the range of milivolt. Sensor output voltage ranging also concern about the pipe diameter \& other liquid properties. Table $1 \&$ Table 2 shows the experimental setup \& miscellaneous components \& ranging of potential independent input variables.

Table 1. Experimental Setup[41]

\begin{tabular}{|c|c|}
\hline Machine/tools & Specification/Description \\
\hline Flow \& Level measurement and Control & Model no. WFT -20-I \\
\hline Flow sensor & Constructed by SL 100 transistor \\
\hline Diameter of PVC pipe & $20 \mathrm{~mm}, 25 \mathrm{~mm} \mathrm{\&} \mathrm{30mm}$ \\
\hline Digital Multimeter & $31 / 2$ \\
\hline Rota meter & Taking the reading of the Flow rate ranging 0-600 Lpm \\
\hline
\end{tabular}

Table 2. Ranges of the process parameters [42]

\begin{tabular}{|c|c|}
\hline Process parameters & Range \\
\hline Output of the Sensor & $210 \mathrm{mv}-285 \mathrm{mv}$ \\
\hline Pipe diameter(mm) & $20 \mathrm{~mm}, 25 \mathrm{~mm} \mathrm{\&} 30 \mathrm{~mm}$ \\
\hline Water conductivity $(\mathrm{W} / \mathrm{m} . \mathrm{k})$ & $606,615 \& 622(\mathrm{~W} / \mathrm{m} . \mathrm{k})$ \\
\hline Water Viscosity & $725.4,779.7 \& 898.2 \mu \mathrm{pas} . \mathrm{sec}$ \\
\hline
\end{tabular}

Table 3.Experimental datasets for liquid flow control process[40]

\begin{tabular}{|l|l|l|l|l|}
\hline Sensor output & Diameter & Conductivity & Viscosity & Flow rate \\
\hline 0.218 & 0.024 & 0.606 & 0.8982 & 0.0008 \\
\hline 0.221 & 0.025 & 0.616 & 0.7797 & 0.0008 \\
\hline 0.225 & 0.025 & 0.616 & 0.8982 & 0.0016 \\
\hline 0.232 & 0.025 & 0.597 & 0.7797 & 0.0016 \\
\hline 0.234 & 0.02 & 0.615 & 0.8982 & 0.0024 \\
\hline 0.237 & 0.027 & 0.622 & 0.7797 & 0.0024 \\
\hline 0.238 & 0.03 & 0.6065 & 0.7254 & 0.0024 \\
\hline 0.239 & 0.025 & 0.616 & 0.8982 & 0.0032 \\
\hline 0.241 & 0.027 & 0.622 & 0.7797 & 0.0032 \\
\hline 0.245 & 0.024 & 0.6065 & 0.7254 & 0.0032 \\
\hline 0.247 & 0.024 & 0.616 & 0.8982 & 0.004 \\
\hline
\end{tabular}




\begin{tabular}{|l|l|l|l|l|}
\hline 0.247 & 0.025 & 0.622 & 0.7797 & 0.004 \\
\hline 0.25 & 0.025 & 0.6065 & 0.7254 & 0.0048 \\
\hline 0.256 & 0.025 & 0.616 & 0.8982 & 0.0048 \\
\hline 0.254 & 0.024 & 0.622 & 0.7797 & 0.0056 \\
\hline 0.259 & 0.03 & 0.606 & 0.7254 & 0.0064 \\
\hline 0.265 & 0.027 & 0.622 & 0.7797 & 0.0072 \\
\hline
\end{tabular}

\section{Mathematical Model of the Problem}

As it is non-linear process system hence flow sensor output voltage of it not only depends upon the change in liquid flow rate but also the other input parameter like liquid properties \& pipe diameter. Here the overall process is divided into two steps: in first stage we use a non-linear power mathematical model namely ANOVA[43] to make a relations between input \& output process variables which followed by the three different computational intelligence tool are used to obtain the optimum flow rate corresponding to the input process variables. In this present case, ANOVA is used to make a power series formulation between liquid flow rate $(F)$ can be expressed in term of sensor output $(E)$, conductivity $(k) \&$ pipe diameter $(D)$ as follows:

$$
F=\mu_{1} \cdot E^{\mu 2} \cdot D^{\mu 3} \cdot k^{\mu 4}
$$

Where, $\mu_{1} \mu_{2} \mu_{3} \& \mu_{4}$ are the coefficients its value can be determined by using some computational intelligence techniques. Now metaheuristics optimization techniques are used to find out the minimum deviation of RMSE by making a difference between calculated \& experimental flow rate of the process. Least value of root mean square error (RMSE) indicates more predicted model. In this work, is represented by Eq. (3).

$$
\operatorname{RMSE}(X)=\sqrt{\frac{\sum_{i=1}^{N} f(E, D, K, X)^{2}}{N}}
$$

Where, $N$ is the number of test data sets $\& X$ is the set of the estimated parameters.

For ANOVA based modelling, the error function $\left(E_{\mathrm{i}}, D_{\mathrm{i}}, \mathrm{k}_{\mathrm{i}}, X\right)$ and set of parameters $X$ can be expressed as

$$
\begin{gathered}
\left(E_{i}, D_{i}, k_{i}, X\right)=\mu_{1} \cdot E^{\mu 2} \cdot D^{\mu 3} \cdot k^{\mu 4}-F \\
X=\left\{\mu_{1}, \mu_{2}, \mu_{3}, \mu_{4}\right\}
\end{gathered}
$$

Littler estimation of the target work gives better arrangement which relates to predominant arrangement of assessed coefficients of ANOVA based model.

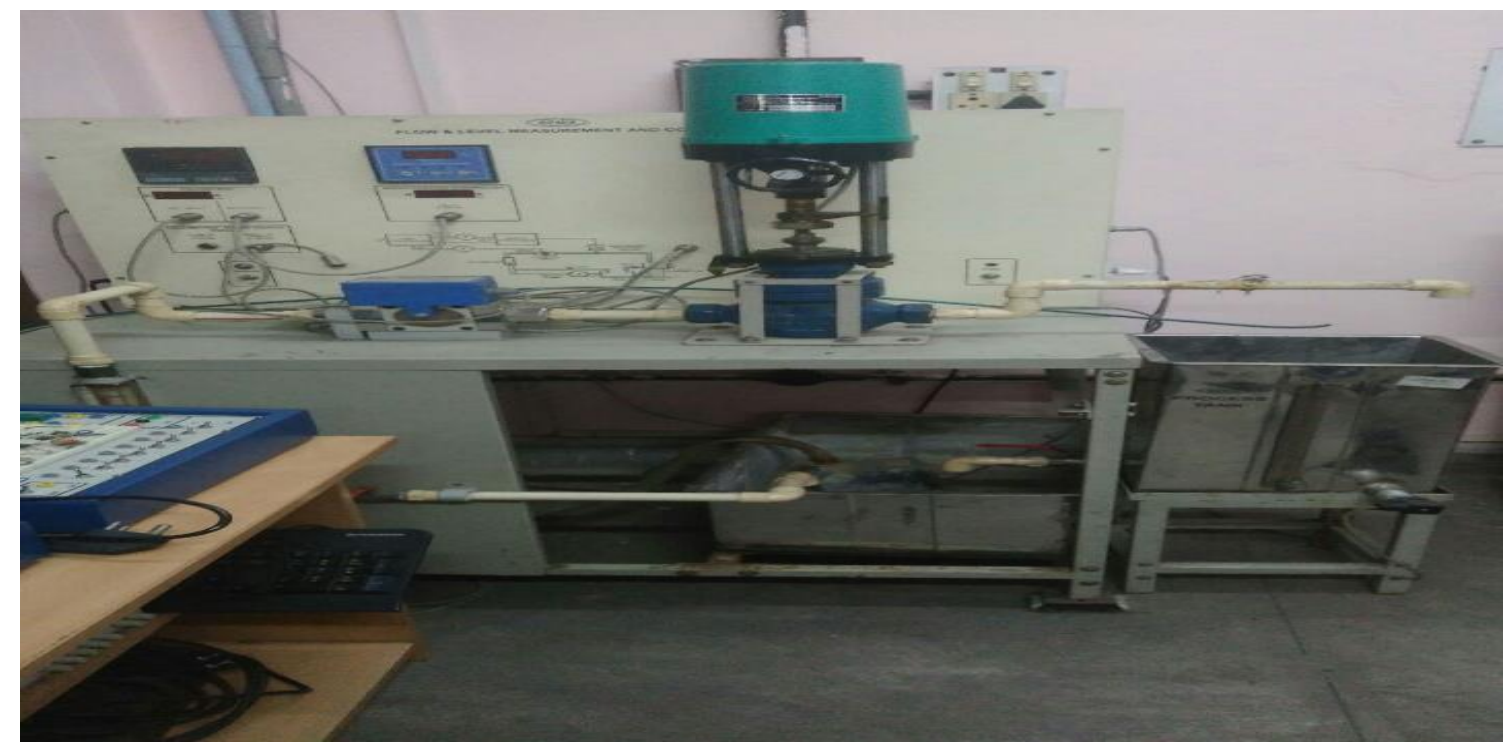

Fig.1. Experimental set up [40] 


\section{A. Improved Grey Wolf Optimization}

Numerous specialists have exhibited a few hybridization variations for heuristic variations. Hybridization can be done by combining the two variants either in low level or elevated level with transfer or co-transformative systems as heterogeneous or homogeneous[41,42,43]. In this, we hybridize Particle Swarm Optimization with Gray Wolf Optimizer calculation utilizing low-level co transformative hybridization. Hybridization is generally low level since we consolidate the functionalities of the two variations. It is co evolutionary in light of the fact that we don't utilize the two variations in a steady progression. In different manners, they run in parallel. It is blended on the grounds that there are two unmistakable variations that are engaged with producing last arrangements of the issues. Based on this adjustment, we improve the capacity of abuse in Particle Swarm Optimization with the capacity of investigation in Gray Wolf Optimizer to deliver the two variations' quality. In HPSOGWO, initial three specialists' position is refreshed in the hunt space by the proposed numerical conditions. Instead of utilizing common scientific conditions; we control the investigation and abuse of the dark fraud space by latency steady. Overall process is shown is shown in Figure 2.

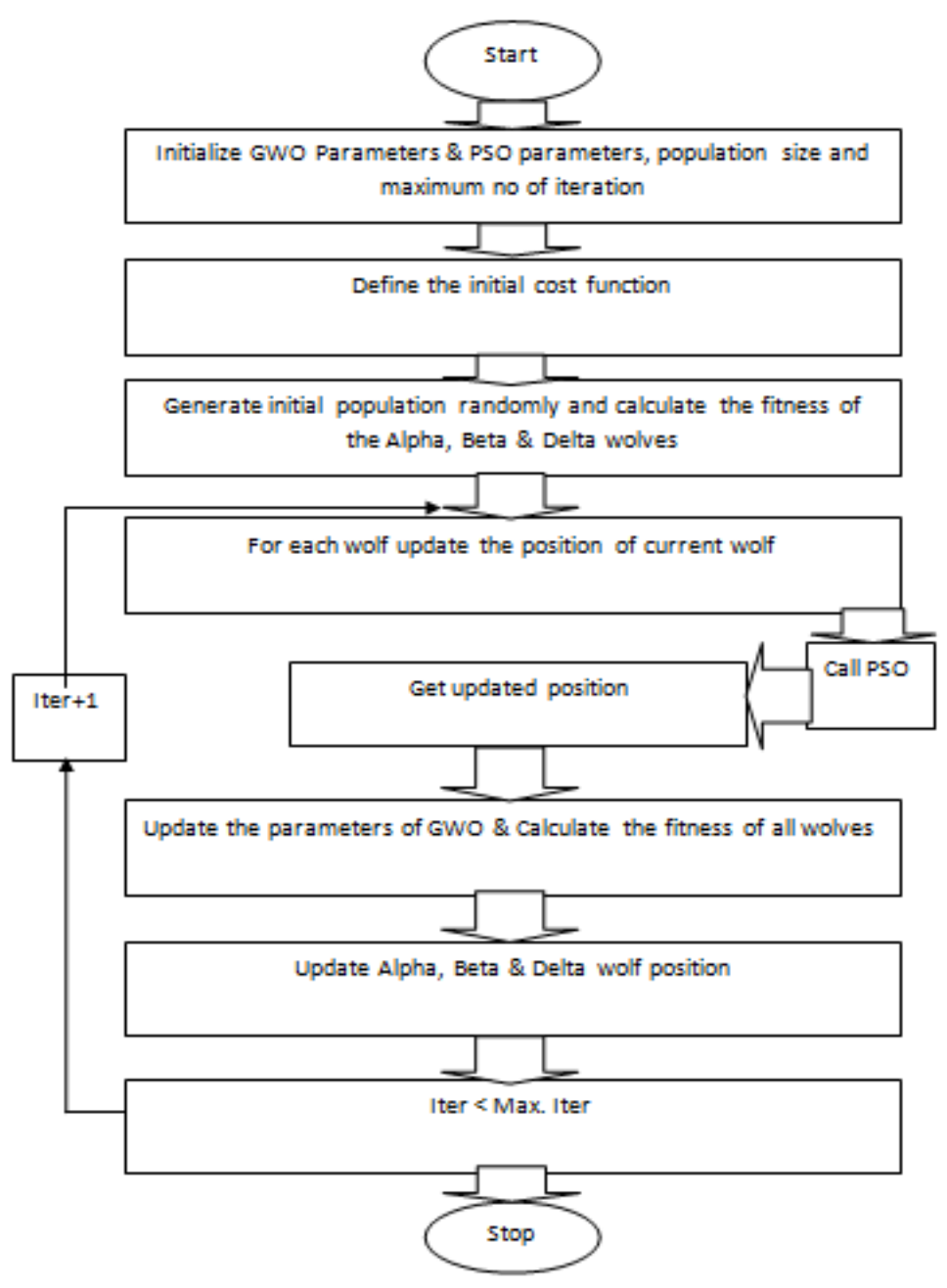

Fig.2. Flowchart of HPSOGWO

Let, the number of wolves is $N$ and the search area is $d$, the position of the ith wolf can be expressed as: $\mathrm{Xi}=\left(\mathrm{X}_{\mathrm{il}}\right.$, $\left.\mathrm{X}_{\mathrm{i} 2}, \mathrm{X}_{\mathrm{i} 3} . \mathrm{X}_{\mathrm{id}}\right)$. to mathematical model the social hierarchy of wolves, the fittest solution is considered as the alpha $(\alpha)$ wolf. Consequently, the second- and third-best solutions are named beta $(\beta)$ and delta $(\delta)$ wolves, respectively. The rest of the candidate solutions are assumed to be omega $(\omega)$ wolves.

Expression for the control parameters of alpha $(\alpha)$, beta $(\beta) \&$ delta $(\partial)$

$$
\overrightarrow{D_{\alpha}}=\left|\overrightarrow{C 1} \cdot \overrightarrow{X_{\alpha}}-\vec{X} * w\right|
$$




$$
\begin{aligned}
& \overrightarrow{D_{\beta}}=\left|\overrightarrow{C 2} \cdot \overrightarrow{X_{\beta}}-\vec{X} * w\right| \\
& \overrightarrow{D_{\delta}}=\left|\overrightarrow{C 3} \cdot \overrightarrow{X_{\delta}}-\vec{X} * w\right|
\end{aligned}
$$

In order to combine PSO and GWO variants, the velocity and updated equation are proposed as follows:

$$
\begin{gathered}
V_{i}^{k+1}=w^{*}\left(V_{i}^{k}+c_{1} r_{1}\left(x_{1}-x_{i}^{k}\right)+c_{2} r_{2}\left(x_{2}-x_{i}^{k}\right)+c_{3} r_{3}\left(x_{3}-x_{i}^{k}\right)\right. \\
x_{i}^{k+1}=x_{i}^{k}+V_{i}^{k+1}
\end{gathered}
$$

In this segment, we present the numerical re-enactment consequences of the hybrid PSOGWO optimization technique on the two previously mentioned benchmark issues. Also, we perform a comparison among HPSOGWO and other two basic optimization techniques: PSO \& GWO, and give a factual investigation of the assessed outcomes. For comparison reason, we have chosen some notable optimization techniques to be specific Gray wolf enhancement (GWO) [33,37-39], and Particle Swarm Optimization (PSO) [46-48]. The parameters set for each algorithm is shown in Table 4.

Table 4 Parameters setting for each Algorithm

\begin{tabular}{|c|c|c|}
\hline PSO & GWO & HPSOGWO \\
\hline Inertia weight may be between 0.9 to 0.4 & Number of wolves is 5 & $\mathrm{c}_{1}=\mathrm{c}_{2}=\mathrm{c}_{3}=0.5$ \\
\hline $\mathrm{C}_{1}=\mathrm{C}_{2}=2$ & Search domain is 36 & $\mathrm{w}=0.5+$ rand $\mathrm{s}() / 2 ;$ and $\mathrm{i}=\epsilon[2,0]$ \\
\hline
\end{tabular}

\section{Result \& Discussion}

For the entire algorithm, we take the maximum number of population is set to 1000. For ANOVA based model, the inquiry space is restricted to 5 for example we have considered 4 dimensional capacity advancement issues looking for ideal estimations of coefficients $\left\{\mu_{1}, \mu_{2}, \mu_{3}, \mu_{4}\right\}$.The scan go for the improvement of fluid stream control process model is set to $[-15,15]$ for the entirety of the coefficients for the two kinds of displaying. Every one of the methods were mimicked utilizing Matlab 2015b in a PC with 4 GB RAM, Intel I center I3 processor and Windows 7 working System. Because of stochastic nature of bio-motivated advancement, they may give distinctive yield depending diverse irregular instatement. The best computational algorithm has been identified based on computational effectiveness and exactness test which are portrayed in this subsection individually.

\section{A. Computational Efficiency Test}

For evaluating the effectiveness of any computational algorithm, computational time is a one of the major factor. In this section we have observed average execution time taken by each algorithm for the given nonlinear specify the computational efficiency. Average execution time for all the three computational algorithm shown in Table 1. From result it is observed that computational efficiency of HPSOGWO based ANOVA model is best.

Table 5. Comparative study on computational time

\begin{tabular}{|c|c|}
\hline Method & Average computational time \\
\hline PSO-ANOVA & $828.7174 \mathrm{sec}$ \\
\hline GWO-ANOVA & $51.243862 \mathrm{sec}$ \\
\hline HPSOGWO-ANOVA & $21.8281 \mathrm{sec}$ \\
\hline
\end{tabular}

\section{B. Convergence Test}

The convergence test was observed by finding the best (minimum) fitness values of the objective function applied in an algorithm at each iteration index. In this research, we execute each algorithm by 20 runs where the number of populations \& maximum iterations sets to $50 \& 5000$ respectively. To graphically represent the convergence test we make a comparative study between PSO, GWO \& HPSOGWO up to 500 iterations. From Figure 3 it is seen that HPSOGWO have has better convergence ability than PSO \& GWO. 
Table 6. Comparative study based on Convergence speed

\begin{tabular}{|c|c|c|c|}
\hline No of Iteration & PSO & GWO & HPSOGWO \\
\hline 100 & 0.0007 & 0.000543 & 0.000443 \\
\hline 1000 & 0.00068 & 0.000537 & 0.00044291 \\
\hline 2000 & 0.000457 & 0.000472 & 0.0005976 \\
\hline 3000 & 0.000452 & 0.000474 & 0.00044269 \\
\hline 4000 & 0.000489 & 0.000442 & 0.000442 \\
\hline 5000 & 0.000465 & 0.000442 & 0.0004337 \\
\hline
\end{tabular}

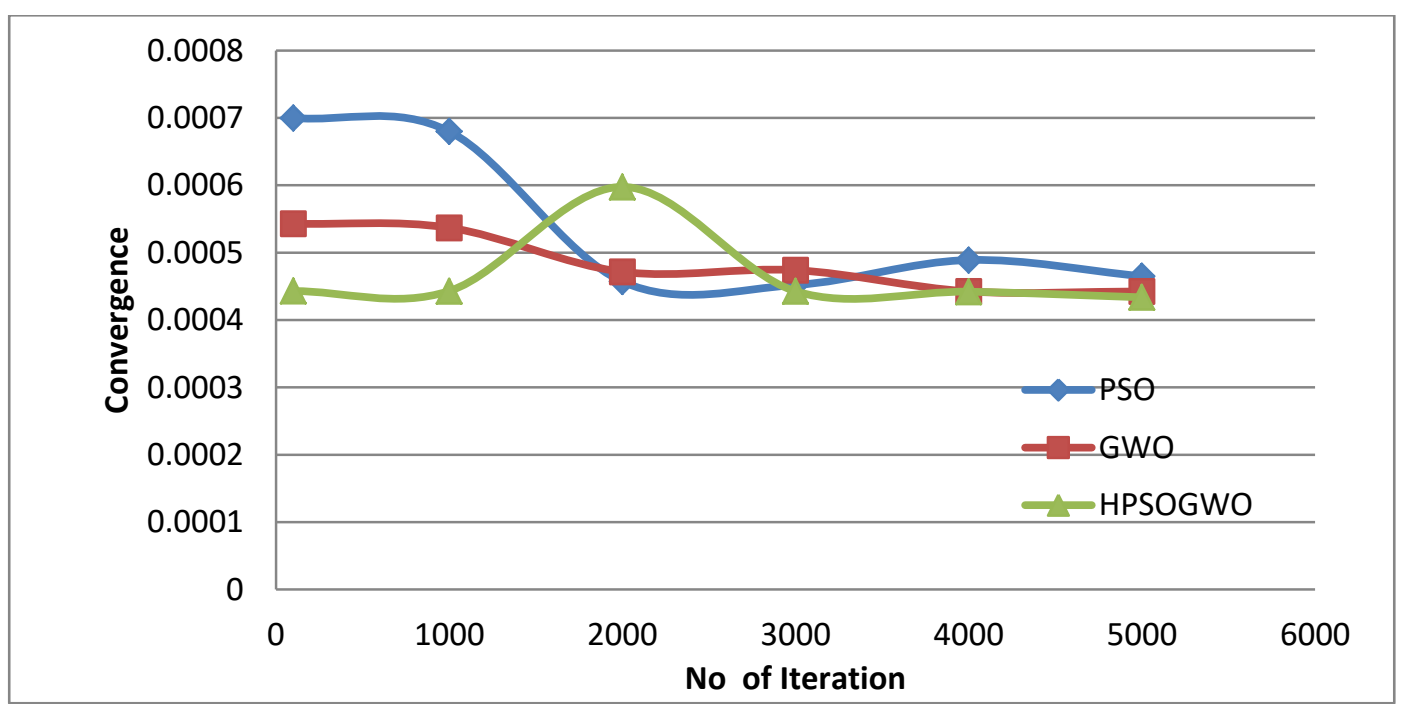

Fig.3.Conergence speed for PSO, GWO \& HPSOGWO

\section{Accuracy Test}

Accuracy test is another parameter to measure the effectiveness of any computational algorithm to observe the prediction capability under different experimental conditions. In this research here we used two different accuracy parameters: IAE \& MAE which are adopted to indicate the error values between the experimental and the simulated current data, defined as Eqn. (7) \& Eqn. (8)

$$
I A E=\mid \text { Fmeasured }- \text { Fcalculated } \mid
$$

Moreover, mean Absolute Error $(\mathrm{M} A E)$ can be defined as:

$$
\mathrm{M} A E=\frac{\sum_{i=1}^{n} I A E_{i}}{n}
$$

Where, $n$ is the number of experimental dataset, Fmeasur \& Fcalculated are represented the experimental and estimated value of liquid flow rate for a given input parameter data sets. However, the best bio inspired algorithm should provide the least RMSE estimated from the tested datasets. The coefficient of the non linear models is obtained from PSO, GWO \& HPSOGWO matlab code shown in Table 7.

Table 7.Estimated optimal parameters by using PSO, GWO \& HPSOGWO

\begin{tabular}{|c|c|c|c|c|c|}
\hline Method & $\mathbf{W}_{\mathbf{1}}$ & $\mathbf{W}_{\mathbf{2}}$ & $\mathbf{W}_{\mathbf{3}}$ & $\mathbf{W}_{\mathbf{4}}$ & $\boldsymbol{\beta}$ \\
\hline PSO & 15 & 10.04667 & -1.0550 & -3.6296 & -0.6204 \\
\hline GWO & 15 & 10.0567 & -1.05728 & -3.3624 & -0.62958 \\
\hline HPSOGWO & 10 & 10.1594 & -1.0604 & -3.9255 & -0.5998 \\
\hline
\end{tabular}


Table 8.Estimated value of Mean absolute error (MAE) in PSO, GWO \& HPSOGWO

\begin{tabular}{|c|c|}
\hline Method & Mean Absolute Error(MAE) \\
\hline PSO & 0.000365 \\
\hline GWO & 0.000365 \\
\hline HPSOGWO & 0.000276 \\
\hline
\end{tabular}

The prediction error can be calculated using Root Mean Square Error (RMSE) which can be defined as follows

$$
\begin{gathered}
R M S E=\sqrt{\frac{1}{m} \sum_{i=1}^{m}\left(\frac{X_{\text {exp }}-X_{C a l}}{X_{\text {exp }}}\right)^{2}} * 100 \% \\
\text { Accuracy }=(100-\text { RMSE }) \%
\end{gathered}
$$

Where $X_{\text {exp }} \& X_{\text {cal }}$ are the experimental \& calculated value and $m$ is number of testing datasets. From Table 8 describes HPSOGWO offers least mean absolute error. It has been also observed from Table 9 that HPSOGWO optimization has least RMSE error \& maximum accuracy. Figure 4 describe the characteristics plot between relative errors vs. no. of instances for PSO, GWO \& HPSOGWO based modeling. From the graph it can be seen that the proposed HPSOGWO having least relative error than PSO \& GWO optimization techniques. So we choose HPSOGWO more suitable in terms of relative error in present process control system.

Table 9.Comparative study based on Root mean square error (RMSE) \& Accuracy

\begin{tabular}{|c|c|c|}
\hline Method & RMSE & Accuracy \\
\hline PSO & 0.04424 & 99.958 \\
\hline GWO & 0.0442 & 99.958 \\
\hline HPSOGWO & 0.0315 & 99.968 \\
\hline
\end{tabular}

From Figure 5 it is seen that HPSOGWO optimization provides better calculated flow rate with respect to experimental flow rate. In every instance both the experimental \& calculated value increases. Figure 6 showing that all the optimization model has minimum deviation when the actual experimental flow rate is ranges from $200 \mathrm{Lpm}$ to 600 Lpm \& HPSOGWO have least deviation with flow rate.

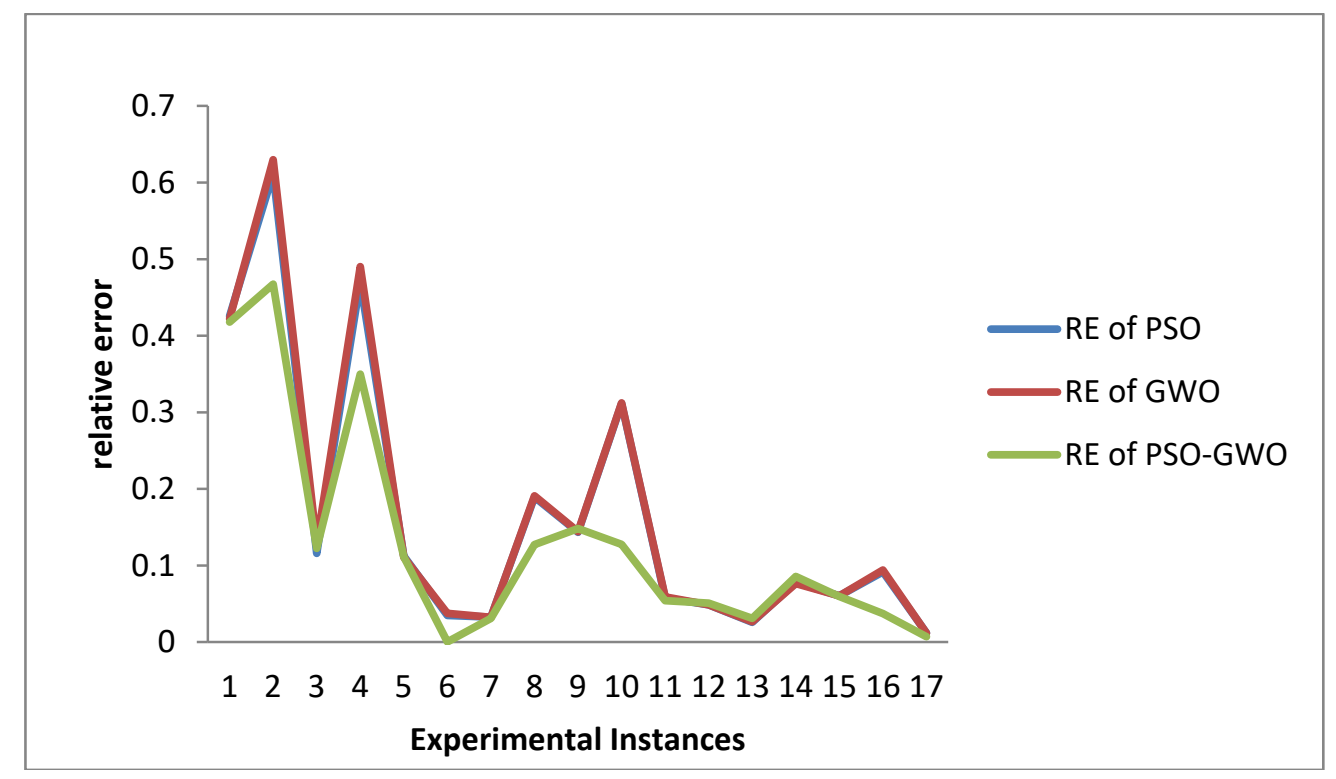

Fig.4. Relative errors for PSO, GWO \& HPSOGWO based modeling 


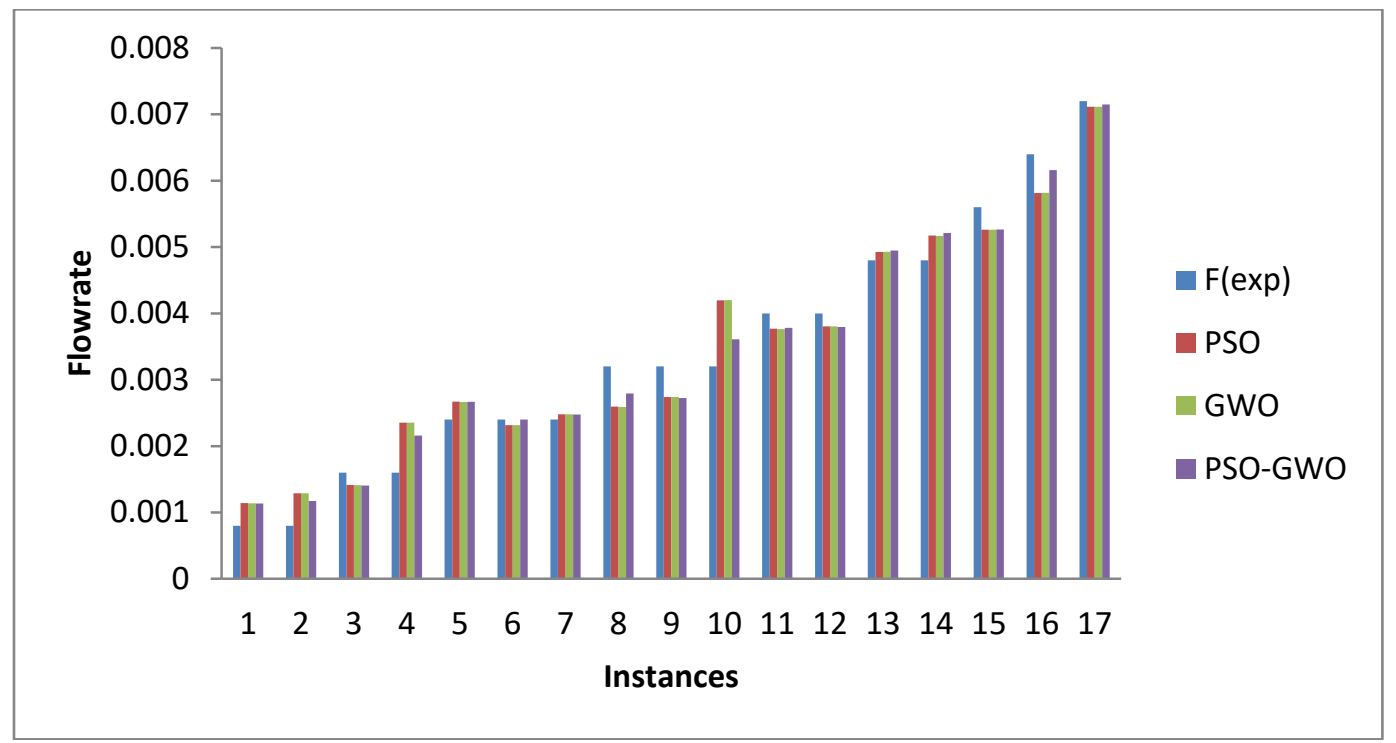

Fig.5. No of Instances V/s experimental data and estimated liquid flow rate

Table 10. Comparison between present study \& previous research

\begin{tabular}{|c|c|c|}
\hline Si No & Model analysis (with reference ) & RMSE \\
\hline $\mathbf{1}$ & FLC & $8 \%$ \\
\hline $\mathbf{2}$ & Neural network model & 2.294 \\
\hline $\mathbf{3}$ & GA-ANN & $1.58 \%$ \\
\hline $\mathbf{4}$ & GA-RSM & $1.03 \%$ \\
\hline $\mathbf{5}$ & GA-ANOVA & $7.4423 \%$ \\
\hline $\mathbf{6}$ & ANFIS & $2.143 \%$ \\
\hline
\end{tabular}

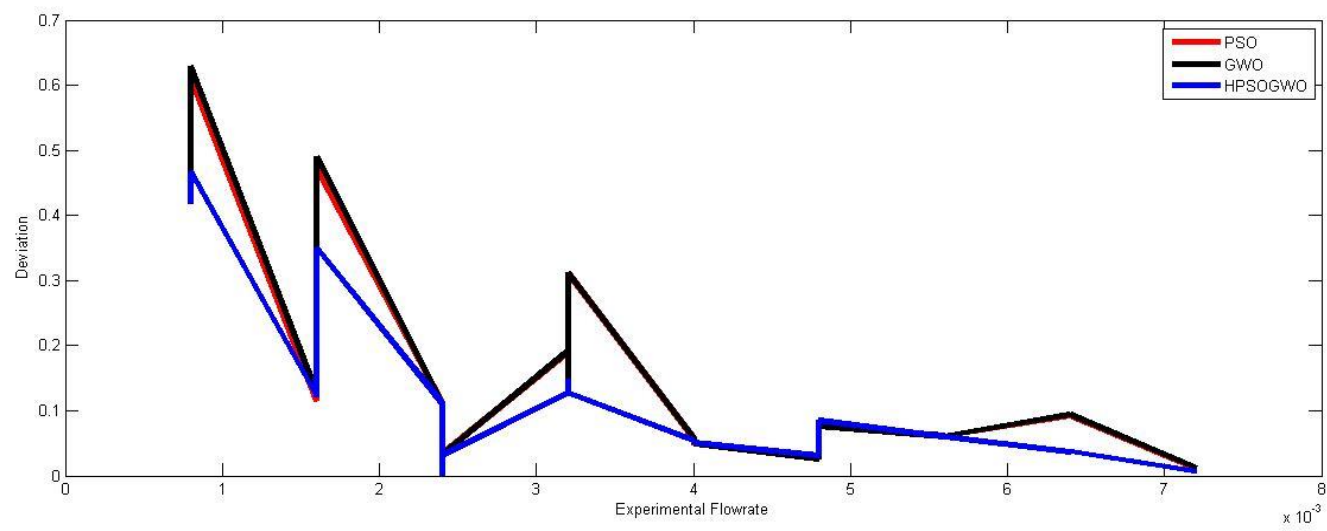

Fig.6. Deviation vs. Experimental flow rate in PSO, GWO \& HPSOGWO

\section{Conclusions}

Demonstrating of efficient liquid flow process control system is a really a fascinating task in modern research. In this research it is seen that experimental liquid flow rate depends upon a number of depended variables like: the sensor output, pipe diameter, liquid (water) viscosity and conductivity. For this examination we take 134 number datasets which contain the combination of independent $\&$ depended variables from lab. Among these informational collections 117 numbers of datasets utilized for creating nonlinear model by ANOVA and rest 17 numbers of datasets is utilized for testing reason.

In following stage we have to discover the ideal estimations of the coefficient of the ANOVA models utilizing some material streamlining approach so anticipated fluid stream rate fit best with the exploratory outcomes. For this reason, we have proposed three different metaheuristic optimization techniques and examined their proficiency for the demonstrating of fluid stream control process. Table 10 shows that the present study provides better accuracy than previously applied intelligent techniques for the same flow control model. 
Numerical recreations are performed and the factual investigation of the outcomes is likewise given. Every one of the outcomes show that the exhibitions of the proposed HPSOGWO beat the PSO and GWO for the greater part of the instances of displaying for fluid stream control process, however significant inconvenience is its computational time. Anyway both the calculations can foresee the fluid stream rate with good exactness like $99.958 \%$ by PSO, $99.958 \%$ by GWO and $99.968 \%$ by HPSOGWO.

This proposed model can be further improved by considering the other independent variables in this liquid fluid control process. In addition to this, further tunings of the metaheuristics are unequivocal to accomplish more proficiency, veracity, union speed, steadiness and achievement rate.

\section{References}

[1] Dutta P, Mandal S, Kumar A. Application of FPA and ANOVA in the optimization of liquid flow control process. RCES 2019;5:7-11. https://doi.org/10.18280/rces.050102.

[2] Dutta P, Mandal S, Kumar A. Comparative study: FPA based response surface methodology \& ANOVA for the parameter optimization in process control. AMA_C 2018;73:23-7. https://doi.org/10.18280/ama_c.730104.

[3] Bera SC, Marik S. Study of a Simple Linearization Technique of p-n-Junction-Type Anemometric Flow Sensor 2012;61:545-52.

[4] Dutta P, Kumar A. Intelligent calibration technique using optimized fuzzy logic controller for ultrasonic flow sensor. MMEP 2017;4:91-4. https://doi.org/10.18280/mmep.040205.

[5] Ashhab M, Al-Salaymeh A. Optimization of hot-wire thermal flow sensor based on a neural net model, 2006. https://doi.org/10.1016/j.applthermaleng.2005.08.004.

[6] Al-Salaymeh A. Optimization of hot-wire thermal flow sensor based on a neural net model. Applied Thermal Engineering 2006;26:948-55.

[7] Santhosh KV, Roy BK. A practically validated intelligent calibration technique using optimized ANN for ultrasonic flow meter. International Journal on Electrical Engineering and Informatics 2015;7:379.

[8] Waller NG, Tellegen A, McDonald RP, Lykken DT. Exploring nonlinear models in personality assessment: Development and preliminary validation of a negative emotionality scale. Journal of Personality 1996;64:545-76.

[9] Dutta P, Mandal S, Kumar A. Comparative study: FPA based response surface methodlogy and ANOVA for the parameter Optimization in Process Control. Advances in Modelling and Analysis C 2018;73:23-7.

[10] Dutta P, Mandal S, Kumar A. Application of FPA and ANOVA in the optimization of liquid flow control process. RCES 2019;5:7-11. https://doi.org/10.18280/rces.050102.

[11] Amemiya T. Non-linear regression models. Handbook of Econometrics 1983;1:333-89.

[12] Dutta P, Kumar A. Design an intelligent flow measurement technique by optimized fuzzy logic controller, 2018. https://doi.org/10.3166/JESA.51.89-107.

[13] Dutta Pijush, Kumar Asok. Study of optimized NN model for liquid flow sensor based on different parameters. Proceeding of international conference on materials, applied physics and engineering, 2018.

[14] Dutta P, Kumar A. Flow sensor Ana- logue:Realtime Predic- tion Analysis using SVM \& KNN, 2018.

[15] Dutta P, Kumar A. Design an intelligent calibration technique using optimized GA-ANN for liquid flow control system. Journal Européen Des Systèmes Automatisés 2017;50:449-70. https://doi.org/10.3166/jesa.50.449-470.

[16] Dutta Pijush, Kumar Asok. Application of an anfis model to optimize the liquid flow rate of a process control system. Chemical Engineering Transactions 2018;71:991-6. https://doi.org/10.3303/CET1871166.

[17] Mandal S, Dutta P, Kumar A. Modeling of liquid flow control process using improved versions of elephant swarm water search algorithm. SN Appl Sci 2019;1:886. https://doi.org/10.1007/s42452-019-0914-5.

[18] Amemiya T. Non-Linear Regression Models//Handbook of Econometrics. Vol. 1/Ed. by Zvi Griliches, Michael D. Intriligator. Amsterdam; New York: North-Holland Pub. Co., 1983. P. 333-389 1983.

[19] Gandomi AH, Yang X-S, Talatahari S, Alavi AH. Metaheuristic applications in structures and infrastructures. Newnes; 2013.

[20] Eberhart RC, Shi Y. Comparing inertia weights and constriction factors in particle swarm optimization. Proceedings of the 2000 congress on evolutionary computation. CEC00 (Cat. No. 00TH8512), vol. 1, IEEE; 2000, p. 84-8.

[21] Dutta P, Agarwala R, Majumder M, Kumar A. PARAMETERS EXTRACTION OF A SINGLE DIODE SOLAR CELL MODEL USING BAT ALGORITHM, FIREFLY ALGORITHM \& CUCKOO SEARCH OPTIMIZATION. Annals of the Faculty of Engineering Hunedoara 2020;18:147-56.

[22] Karaboga D, Basturk B. A powerful and efficient algorithm for numerical function optimization: artificial bee colony (ABC) algorithm. Journal of Global Optimization 2007;39:459-71.

[23] Yang X-S. Firefly algorithm, stochastic test functions and design optimisation. International Journal of Bio-Inspired Computation 2010;2:78-84.

[24] Yang X-S. Flower pollination algorithm for global optimization. International conference on unconventional computing and natural computation, Springer; 2012, p. 240-9.

[25] Dorigo M, Maniezzo V, Colorni A. Ant system: optimization by a colony of cooperating agents. IEEE Transactions on Systems, Man, and Cybernetics, Part B (Cybernetics) 1996;26:29-41.

[26] Yang X-S, Deb S. Engineering optimisation by cuckoo search. International Journal of Mathematical Modelling and Numerical Optimisation 2010;1:330-43.

[27] Mandal S, Dutta P, Kumar A. Modeling of liquid flow control process using improved versions of elephant swarm water search algorithm. SN Applied Sciences 2019;1:1-16.

[28] Dutta P, Biswas SK, Biswas S, Majumder M. Parametric optimization of Solar Parabolic Collector using metaheuristic Optimization. Computational Intelligence and Machine Learning 2021;2:26-32. 
[29] Onyezewe A, Kana AF, Abdullahi F, Abdulsalami A. An Enhanced Adaptive k-Nearest Neighbor Classifier Using Simulated Annealing. International Journal of Intelligent Systems and Applications 2021;13:34-44. https://doi.org/10.5815/ijisa.2021.01.03.

[30] Messaoudi A, Belkacemi M. Optimal Power Flow Solution using Efficient Sine Cosine Optimization Algorithm. International Journal of Intelligent Systems and Applications 2020;12:34-43. https://doi.org/10.5815/ijisa.2020.02.04.

[31] Sharma D, Dubey H, Pandit M. Single and Multi-Area Optimal Dispatch by Modified Salp Swarm Algorithm. International Journal of Intelligent Systems and Applications 2020;12:18-26. https://doi.org/10.5815/ijisa.2020.03.03.

[32] Wolpert DH. The Implications of the No-Free-Lunch Theorems for Meta-induction. ArXiv Preprint ArXiv:210311956 2021.

[33] Mirjalili S, Mirjalili SM, Lewis A. Grey Wolf Optimizer. Advances in Engineering Software 2014;69:46-61. https://doi.org/10.1016/j.advengsoft.2013.12.007.

[34] Wen Long, Songjin Xu. A novel grey wolf optimizer for global optimization problems. 2016 IEEE Advanced Information Management, Communicates, Electronic and Automation Control Conference (IMCEC), 2016, p. $1266-70$. https://doi.org/10.1109/IMCEC.2016.7867415.

[35] Zhu A, Xu C, Li Z, Wu J, Liu Z. Hybridizing grey wolf optimization with differential evolution for global optimization and test scheduling for 3D stacked SoC. Journal of Systems Engineering and Electronics 2015;26:317-28. https://doi.org/10.1109/JSEE.2015.00037.

[36] Singh N, Singh S. A Modified Mean Gray Wolf Optimization Approach for Benchmark and Biomedical Problems. Evol Bioinform Online 2017; 13:1176934317729413. https://doi.org/10.1177/1176934317729413.

[37] Li Q, Chen H, Huang H, Zhao X, Cai Z, Tong C, et al. An Enhanced Grey Wolf Optimization Based Feature Selection Wrapped Kernel Extreme Learning Machine for Medical Diagnosis. Computational and Mathematical Methods in Medicine 2017;2017:9512741. https://doi.org/10.1155/2017/9512741.

[38] Emary E, Zawbaa HM, Grosan C. Experienced Gray Wolf Optimization Through Reinforcement Learning and Neural Networks - IEEE Journals \& Magazine. IEEE Transactions on Neural Networks and Learning Systems, 2017.

[39] Zhang S, Zhou Y. Grey Wolf Optimizer Based on Powell Local Optimization Method for Clustering Analysis. Discrete Dynamics in Nature and Society 2015;2015:481360. https://doi.org/10.1155/2015/481360.

[40] Dutta P, Kumar A. Modeling and Optimization of a Liquid Flow Process using an Artificial Neural Network-Based Flower Pollination Algorithm. Journal of Intelligent Systems 2019;29:787-98. https://doi.org/10.1515/jisys-2018-0206.

[41] Dutta P, Kumar A. Modelling of Liquid Flow control system Using Optimized Genetic Algorithm. Statistics, Optimization \& Information Computing 2020;8:565-82. https://doi.org/10.19139/soic-2310-5070-618.

[42] Dutta P, Kumar A. Modelling of Liquid Flow control system Using Optimized Genetic Algorithm. Statistics, Optimization \& Information Computing 2020;8:565-82.

[43] Glantz S, Slinker B, Neilands TB. Primer of Applied Regression \& Analysis of Variance 3E. 2016.

[44] Şenel FA, Gökçe F, Yüksel AS, Yiğit T. A novel hybrid PSO-GWO algorithm for optimization problems. Engineering with Computers 2019;35:1359-73.

[45] Mishra AK, Das SR, Ray PK, Mallick RK, Mohanty A, Mishra DK. PSO-GWO Optimized Fractional Order PID Based Hybrid Shunt Active Power Filter for Power Quality Improvements. IEEE Access 2020;8:74497-512.

[46] Xiang Z, Ji D, Zhang H, Wu H, Li Y. A simple PID-based strategy for particle swarm optimization algorithm. Information Sciences 2019;502:558-74.

[47] Sangeetha AL, Bharathi N, Ganesh AB, Radhakrishnan TK. Particle swarm optimization tuned cascade control system in an Internet of Things (IoT) environment. Measurement 2018;117:80-9.

[48] Wang D, Tan D, Liu L. Particle swarm optimization algorithm: an overview. Soft Computing 2018;22:387-408.

\section{Authors' Profiles}

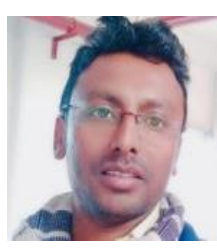

Prof. Pijush Dutta is currently working as an Assistant Professor in Department of Electronics \& communication Engineering, Global Institute of Management \& Technology, India. He received his B Tech \& M Tech from West Bengal University of Technology, India in 2007 \& 2012 respectively. Presently he persuing his $\mathrm{Ph}$ D from Mewar University, India. Till now he published more than 30 research journal, Conference \& Book chapter, 2 author book \& 11 internal \& national Patent. His research interests are Optimization, intelligent system , Internet of Things, Machine Learning etc.

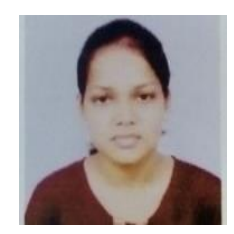

Mrs. Madhurima Majumder is currently acting as a lecturer in Department of electrical \& Electronics Engineering Department, Mirmadan Mohanlal Government Polytechnic Institute, India. She received her B.Tech from MAKAUT, India in 2019. Presently she persuing M Tech from MAKAUT. Till now she published 3 research papers in a reputated journal. Her research interests are Internet of Things \& Artificial Intelligence. 


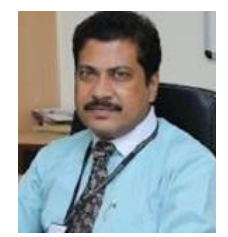

Prof. (Dr.) Asok Kumar is currently working as a Dean of Student welfare Department, Vidya Sagar University, India. He received his M.Tech from Calcutta University \& Ph D Degree from Jadavpur University in 1999 \& 2007 respectively. Till now he published more than 70 reputated journal, conference, \& book chapter and 2 author book. His research interests are networking, coding techniques, Intelligent system etc.

How to cite this paper: Pijush Dutta, Madhurima Majumder, Asok Kumar, " An Improved Grey Wolf Optimization Algorithm for Liquid flow Control System", International Journal of Engineering and Manufacturing (IJEM), Vol.11, No.4, pp. 10-21, 2021. DOI: 10.5815/ijem.2021.04.02 